\title{
The KMOS Galaxy Clusters Project
}

\author{
Roger L. Davies ${ }^{1}$, A. Beifiori ${ }^{2}$, R. Bender ${ }^{2}$, M. Cappellari $^{1}$, J. Chan $^{2}$,

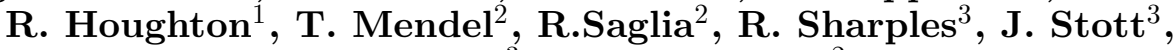 \\ R. Smith ${ }^{3}$ and D. Wilman ${ }^{2}$ \\ ${ }^{1}$ Sub-department of Astrophysics, Department of Physics, University of Oxford, \\ Denys Wilkinson Building, Keble Road, Oxford OX1 3RH. email: rld@astro.ox.ac.uk \\ ${ }^{2}$ Max Planck Institut für Extraterrestrische Physik \& University Observatory of the \\ Ludwig-Maximilians-University, Munich. \\ ${ }^{3}$ Department of Physics \& Astronomy, Durham University.
}

\begin{abstract}
KMOS is a cryogenic infrared spectrograph fed by twentyfour deployable integral field units that patrol a 7.2 arcminute diameter field of view at the Nasmyth focus of the ESO VLT. It is well suited to the study of galaxy clusters at $1<\mathrm{z}<2$ where the well understood features in the restframe V-band are shifted into the KMOS spectral bands. Coupled with HST imagining, KMOS offers a window on the critical epoch for galaxy evolution, 7-10 Gyrs ago, when the key properties of cluster galaxies were established. We aim to investigate the size, mass, morphology and star formation history of galaxies in the clusters. Here we describe the instrument, discuss the status of the observations and report some preliminary results.
\end{abstract}

Keywords. galaxies: elliptical and lenticular, galaxies: formation, galaxies: clusters

\section{Scientific motivation}

'Red and dead' quiescent early type galaxies are abundant in local rich clusters of galaxies where star forming, spiral galaxies are rare (Dressler 1980). At $\mathrm{z}=0.5$ the fraction of star forming disk galaxies is higher, (Dressler et al. 1997). These results are intrepreted in terms of the transformation of spiral to S0 galaxies over the 4.5Gyr interval. We thus expect the diversity of the Hubble sequence to be established at $0.5<\mathrm{z}<2$ during the peak epoch of star formation (Madau et al. 1996, 1998). Studying high density environments at these redshifts is imperative to reveal the mechanisms that quench star formation and generate the distribution of morphological types we see in local clusters today. Mechanisms that have been suggested for quenching star formation include ram pressure stripping, (Gunn \& Gott 1972), harassment, (Moore et al. 1996), and strangulation (Larson et al. 1980).

The evolution of early-type galaxies can be studied through measuring their scaling relations, such as the Fundamental Plane, as a function of redshift. Several studies have revealed that massive ETGs are in place at z 2 (e.g. Cimatti et al. 2004) but with smaller sizes (e.g. Trujillo et al. 2007; Newman et al. 2012) and higher stellar velocity dispersions (e.g. Cappellari et al. 2009; Saglia et al. 2010; Toft et al. 2012; Onodera et al. 2012; van de Sande et al. 2013) compared to local counterparts. However, these authors disagree on the degree of evolution in size (Mancini et al. 2010; Szomoru et al. 2011) and velocity dispersion (van Dokkum et al. 2009; Cappellari et al. 2009; Onodera et al. 2012), which prevents us from identifying the true cause of these changes.

Furthermore locally we observe that the stars in the most massive galaxies formed at the highest redshifts (Thomas et al. 2005) and, according to $[\alpha / \mathrm{Fe}]$ excess, over short timescales from high metallicity gas. This shift of active star formation from high mass to low mass galaxies is known as 'downsizing' (Cowie et al. 1996). Although the dynamical 

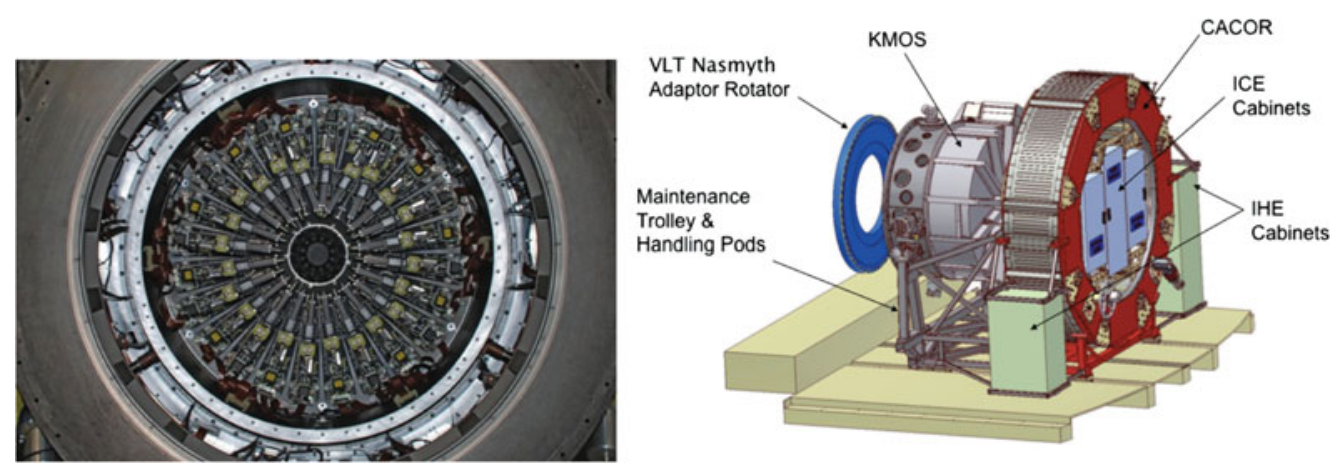

Figure 1. The left hand image shows the 24 pickoff arms of KMOS in their retracted positions. The right hand schematic illustrates the position of KMOS on the Nasmyth platform of the VLT.

$\mathrm{M} / \mathrm{L}$ evolution of the Fundamental Plane up to $\mathrm{z} \sim 1$ is consistent with passive evolution (Barr et al. 2006; Jørgensen et al. 2006; van Dokkum \& van der Marel 2007; Saglia et al. 2010), the presence of downsizing in the FP is strongly contested (Fritz et al. 2009; Holden et al. 2010).

It has been proposed that the evolution of ETGs is driven by a combination of their merging history and mass-to-light evolution (Toft et al. 2012). Many mechanisms have been proposed such as galaxy growth by major/minor and/or dry/wet mergers and even secular-like "puffing" via AGN feedback (e.g. Hopkins et al. 2009, 2010; Oser et al. 2012; Hilz et al. 2012). Understanding which of these mechanisms drives the evolution of the scaling relations to low-z relies on measuring sizes and velocity dispersions for a 'fair' sample of galaxies at high-z. To date this has not proved possible because, although deep, high-quality imaging data are available for numerous $\mathrm{z}>1$ clusters, current $\mathrm{z} \sim 1$ spectroscopic studies have sampled only the brightest and most massive galaxies in a few clusters, rarely extending below $10^{11} M_{\odot}$ and typically selecting only a few galaxies per cluster (van Dokkum \& van der Marel 2007)). Dynamical studies at z $>1.3$ only exist for few tens of the brightest objects (van Dokkum et al. 2009; Toft et al. 2012; van de Sande et al. 2013; Belli et al. 2014), or for stacked spectra (Cappellari et al. 2009).

With the advent of KMOS many of these impediments to the study of high redshift clusters have been removed. The factor of 24 multiplexing allows more than an order-ofmagnitude increase in observing efficiency, the IFU eliminates slit losses and the latest NIR detectors are as efficient as CCDs beyond $0.8 \mu \mathrm{m}$, allowing us to observe at $\mathrm{z}>1$. We are investing significant GTO resources to undertake a deep spectroscopic survey of large numbers of galaxies $(>80)$, which heretofore would not be feasible. Using this sample we aim to (1) exploit the FP and dynamical M/L studies at $\mathrm{z} \sim 1.5$ to constrain formation ages; (2) measure the evolution in size and velocity dispersion of a large, representative sample of individual galaxies, to robustly distinguish between different merger history models, (3) use well-understood models of the optical indices to measure stellar ages and metallicities at a time where differences are pronounced and free from low-z residual SF (Kaviraj et al. 2007, 2008) and (4) characterise the evolution of age, metallicity and size along the FP (Graves et al. 2009; Graves \& Faber 2010; Saglia et al. 2010; Toft et al. 2012; Bezanson et al. 2013; van de Sande et al. 2014) to reveal how ETGs are assembled in the cluster environment. 


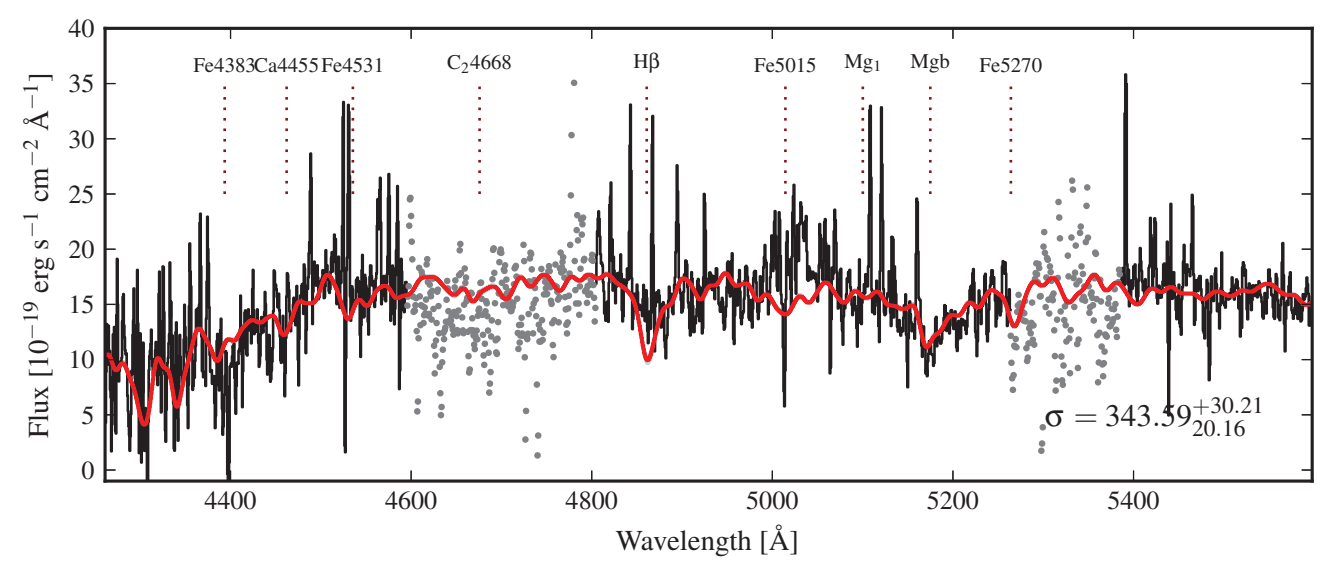

Figure 2. A spectrum of the $\mathrm{H}=21$ galaxy 576 from the $\mathrm{z}=1.39$ cluster XMMU J2235-2557. The on-source exposure time is 11 hours. The spectrum is extracted in a 1.5 arcsecond diameter aperture. The red line superimposed on the spectrum is a fit, using the MILES stellar library (Sánchez-Blázquez et al. 2006), indicating a velocity dispersion of $344 \mathrm{kms}^{-1}$.

\section{The KMOS instrument}

KMOS is an infrared instrument comprising three spectrometers in a single cryostat each fed from eight image sliced integral field units. Each IFU has $14 \times 14$ spaxels each spanning 2.8 arcseconds square on the sky. They can be deployed within a 7.2 arcminute field of view at the Nasmyth focus of the VLT. The instrument therefore has the potential to observe 24 galaxies simultaneously with an array of 196 spaxels on each galaxy. The 24 retracted arms are shown in the focal plane in Figure 1 where the layout of the instrument on the Nasmyth platform is also shown. The spectrometers provide wavelength coverage from $0.8-2.5 \mu \mathrm{m}$ in each of IZ, YJ, $\mathrm{H} \& \mathrm{~K}$ at a resolution of 3300-3800 to facilitate accurate sky subtraction in the wavelength ranges dominated by strong sky emission lines. The efficiency of the spectrograph is $>20 \%$ in YJ and $>30 \%$ in $\mathrm{H}$ and K. More details of the technical performance can be found in (Sharples et al. 2014) and some early commissioning and science verification results are presented in (Sharples et al. 2013). The instrument was commissioned during 2013 and we started our cluster observations, which use the YJ bands, in October 2013.

\section{The selection of clusters and galaxies}

We selected three clusters for study: XMMU J2235-2257 ( $\mathrm{z}=1.39)$, XMMXCSJ2215.9-

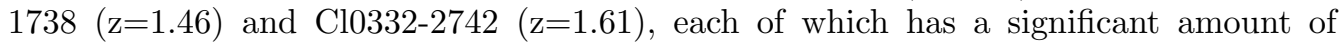
archive data available and a rich enough ETG population to address our science goals. As a backup for when the wind is from the north, or in less optimal observing conditions we selected RCS234526-3632.6 ( $\mathrm{z}=1.04$ ) which has the $\mathrm{CaH}+\mathrm{K}, \mathrm{H} \gamma$, and V-band indices in the KMOS IZ band. Multiband HST photometry is available for all clusters including existing or upcoming WFC3 IR imaging from our HST Cycle 22 program. This is crucial for measuring the sizes of galaxies, mapping the distribution of stellar mass and measuring their structural parameters (which are necessary to construct the Fundamental Plane in stellar mass and light), and determining morphologies. Moreover, deep ground based imaging allows us to identify samples of galaxies on the red sequence and fit spectral energy distributions. Galaxies that have spectroscopically confirmed cluster membership increases our selection efficiency. At $\mathrm{z} \sim 1.4 \& \mathrm{z}=1.6$ the key absorption features (Balmer, 
Mg \& Fe lines) are relatively uncontaminated by strong sky emission or telluric absorption. The KMOS patrol field is well matched to the extent of the clusters on the sky and the IFUs match the sizes of the galaxies. Our cluster sample spans a range of cluster mass, temperature, and morphology, so we can study the clusters through comparison with simulations.

Individual galaxies were initially selected from pre-existing (spectroscopic) membership catalogs, and then from the red sequence below $\mathrm{J}(\mathrm{AB})<22.5$ mag or the equivalent in $\mathrm{H}$ band. We fill unallocated IFUs with blue star-forming objects: confirmed cluster members first, then above the mass limit imposed by the red sequence magnitude limit.

\section{Early results}

Figure 2 shows the spectrum of one of the brighest galaxies, \#576, in XMMUJ22352557 summed over an aperture of diameter 1.5 arcseconds obtained after 11 hours of onsource integration. Absorption features of associated with the $\mathrm{MgH}$ bandhead at $5200 \AA$ are apparent as is $\mathrm{H} \beta$. The model spectrum overplotted is from the MILES library broadened to a velocity dispersion of $344 \mathrm{kms}^{-1}$. Although it is possible to derive velocities and dispersions for the brightest galaxies in the cluster it will be necessary to stack spectra for the faintest galaxies. As yet we do not have sufficient high quality spectra to explore the Fundamental Plane.

The power of the IFU to reveal physical structure that is hard to extract from a slit spectrum is illustrated in Figure 3, which shows, on the left, HST WFC3 F110W images of three galaxies in XMMUJ2235-2557 and on the right the stellar continuum, shown in red, and in two cases emission from the ionised gas ([OIII] 5007 $\AA$ ), shown in green. Between them is the ESO/VLT HAWK-I J \& K composite image of the cluster showing the location of the three galaxies. All three are some way from the centre of the cluster and outside the region of X-ray emission shown in (Mullis et al. 2005). For the two galaxies showing emission, the ionised gas is offset significantly from the stellar continuum indicating that this ionised gas is either flowing out of the galaxy or swept back by the motion of the galaxy through the intracluster medium, even at the low densities implied by the X-ray emission. We are investigating these possibilities further.

\section{Conclusions}

The multi-IFU infrared spectrometer, KMOS, has been brought into operation on the ESO VLT and we are using it to study the evolution of galaxies in rich clusters with $1<\mathrm{z}<2$. At these redshifts the well understood V-band absorption features are shifted into the infrared and are detected in the individual spectra of the brightest galaxies. Coupled with the HST imaging available for the clusters we will study the evolution of the ETG scaling relations and through that the mass-to-light ration evolution. The multiple integral field units enable us to examine the spatial distribution of emission at different wavelengths and we have shown that the emission of [OIII] in two galaxies is significantly offset from the centre of the galaxy potential, even in galaxies that the X-ray maps indicate are in regions of low intracluster medium density.

\section{Acknowledgements}

This work was supported by the Astrophysics at Oxford grants (ST/H002456/1 and $\mathrm{ST} / \mathrm{K} 00106 \mathrm{X} / 1$ ) as well as visitors grant (ST/H504862/1) from the UK Science and Technology Facilities Council. RLD acknowledges travel and computer grants from Christ 

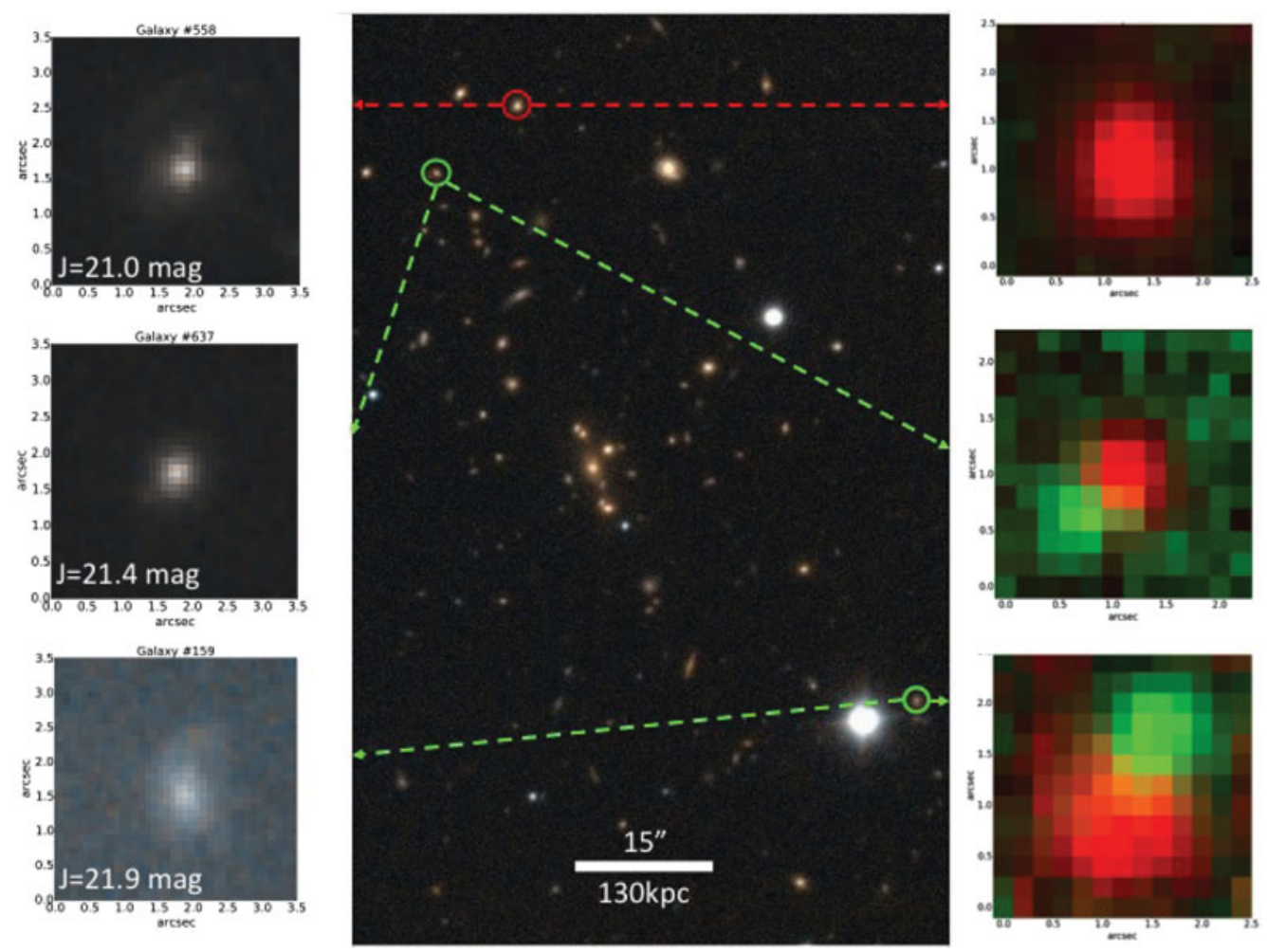

Figure 3. The central panel shows the ESO/VLT HAWK-I J \& K composite image of the $\mathrm{z}=$ 1.39 cluster XMMUJ2235-2557. On the left are HST WFC3 F110W thumbnail images of three galaxies with $\mathrm{J}=21.0$ to 21.9. To the right are the same galaxies but shown in two band passes, (i) in the J-band stellar continuum shown in red and (ii) the emission from [OIII] $5007 \AA$ shown in green. The lower two galaxies exhibit emission significantly offset from the stellar continuum indicating that the ionised gas has been disturbed. Note that the 'stretch' for the emission line (green) images on the right has been chosen to emphasise the presence of emission.

Church, Oxford. The KMOS project has been supported by the Verbundforschung of the German Ministry for Education and Reseach (BMBF), project number 05A11WMA."

\section{References}

Barr, J., Jørgensen, I., Chiboucas, K., Davies, R., \& Bergmann, M. 2006, ApJ, 649, L1

Belli, S., Newman, A. B., \& Ellis, R. S. 2014, ApJ, 783, 117

Bezanson, R., van Dokkum, P. G., van de Sande, J., et al. 2013, ApJ, 779, L21

Cappellari, M., di Serego Alighieri, S., Cimatti, A., et al. 2009, ApJ, 704, L34

Cimatti, A., Daddi, E., Renzini, A., et al. 2004, Nature, 430, 184

Cowie, L. L., Songaila, A., Hu, E. M., \& Cohen, J. G. 1996, AJ, 112, 839

Dressler, A. 1980, ApJ, 236, 351

Dressler, A., Oemler, A., Jr., Couch, W. J., et al. 1997, ApJ, 490, 577

Fritz, A., Böhm, A., \& Ziegler, B. L. 2009, MNRAS, 393, 1467

Graves, G. J., Faber, S. M., \& Schiavon, R. P. 2009, ApJ, 698, 1590

Graves, G. J. \& Faber, S. M. 2010, ApJ, 717, 803

Gunn, J. E. \& Gott, J. R., III 1972, ApJ, 176, 1

Hilz, M., Naab, T., Ostriker, J. P., et al. 2012, MNRAS, 425, 3119

Holden, B. P., van der Wel, A., Kelson, D. D., Franx, M., \& Illingworth, G. D. 2010, ApJ, 724, 714 
Hopkins, P. F., Somerville, R. S., Cox, T. J., et al. 2009, MNRAS, 397, 802

Hopkins, P. F., Bundy, K., Croton, D., et al. 2010, ApJ, 715, 202

Jørgensen, I., Chiboucas, K., Flint, K., et al. 2006, ApJ, 639, L9

Kaviraj, S., Rey, S.-C., Rich, R. M., Yoon, S.-J., \& Yi, S. K. 2007, MNRAS, 381, L74

Kaviraj, S., Khochfar, S., Schawinski, K., et al. 2008, MNRAS, 388, 67

Larson, R. B., Tinsley, B. M., \& Caldwell, C. N. 1980, ApJ, 237, 692

Madau, P., Ferguson, H. C., Dickinson, M. E., et al. 1996, MNRAS, 283, 1388

Madau, P., Pozzetti, L., \& Dickinson, M. 1998, ApJ, 498, 106

Mancini, C., Daddi, E., Renzini, A., et al. 2010, MNRAS, 401, 933

Moore, B., Katz, N., Lake, G., Dressler, A., \& Oemler, A. 1996, Nature, 379, 613

Mullis, C. R., Rosati, P., Lamer, G., et al. 2005, ApJ, 623, L85

Newman, A. B., Ellis, R. S., Bundy, K., \& Treu, T. 2012, ApJ, 746, 162

Onodera, M., Renzini, A., Carollo, M., et al. 2012, ApJ, 755, 26

Oser, L., Naab, T., Ostriker, J. P., \& Johansson, P. H. 2012, ApJ, 744, 63

Saglia, R. P., Sánchez-Blázquez, P., Bender, R., et al. 2010, A\&̊A, 524, A6

Sánchez-Blázquez, P., Peletier, R. F., Jiménez-Vicente, J., et al. 2006, MNRAS, 371, 703

Sharples, R., Bender, R., Agudo Berbel, A., et al. 2013, The Messenger, 151, 21

Sharples, R., Bender, R., Agudo Berbel, A., et al. 2014, Proc. SPIE, 9147, 91470W

Szomoru, D., Franx, M., Bouwens, R. J., et al. 2011, ApJ, 735, L22

Thomas, D., Maraston, C., Bender, R., \& Mendes de Oliveira, C. 2005, ApJ, 621, 673

Toft, S., Gallazzi, A., Zirm, A., et al. 2012, ApJ, 754, 3

Trujillo, I., Conselice, C. J., Bundy, K., et al. 2007, MNRAS, 382, 109

van de Sande, J., Kriek, M., Franx, M., et al. 2013, ApJ, 771, 85

van de Sande, J., Kriek, M., Franx, M., Bezanson, R., \& van Dokkum, P. G. 2014, ApJ, 793, L31

van Dokkum, P. G. \& van der Marel, R. P. 2007, ApJ, 655, 30

van Dokkum, P. G., Kriek, M., \& Franx, M. 2009, Nature, 460, 717 